\title{
TUNING SECTION 2 OF THE LEDA CCDTL*
}

\author{
L.J. Rybarcyk ${ }^{\dagger}$, R.L. Wood, P.O. Leslie, R. Valdiviez, Los Alamos National Laboratory; \\ M.D. Cole, Advanced Energy Systems; J.M. Potter, JP Accelerator Works, USA
}

\begin{abstract}
As part of the Low-Energy Demonstration Accelerator (LEDA) portion of the Advanced Accelerator Applications (AAA) project we have fabricated and will perform high-power RF tests on Section 2 of the 700MHz Coupled-Cavity Drift-Tube Linac (CCDTL). This CCDTL section contains six, two-gap accelerating cells. This portion of the CCDTL was designed to accelerate the 100-mA, LEDA RFQ proton beam to $7.3 \mathrm{MeV}$. This paper reports on the process and results of tuning Section 2 leading up to high-power RF testing.
\end{abstract}

\section{DESCRIPTION OF SECTION 2}

Section 2 of the LEDA CCDTL is comprised of six two-gap accelerating (AC) (cell no. 7-12) and five transverse coupling (TC) cavities. Each end-AC cell also has half of a longitudinal coupling (LC) cell attached. The TC and LC cell designations refer to the orientation of their axis with respect to the Section-2 beam axis. Bridge coupling (BC) cavities that adjoin LC half-cells are then used to combine adjacent sections together. A cut-away view of Section 2 is shown in Figure 1. The RF properties

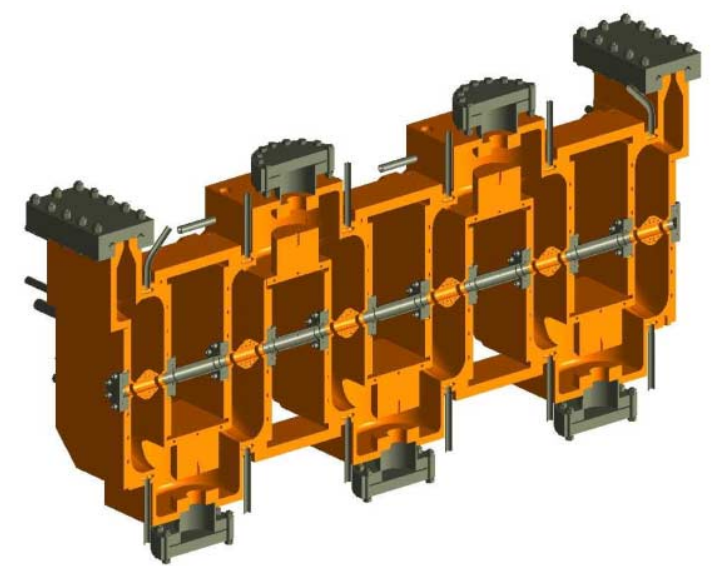

Figure 1: Cut-away view of LEDA CCDTL Section 2.

of the CCDTL structure are explained elsewhere [1]. Each $\mathrm{AC}$ cavity is built up from two half cells joined to a ring that supports the drift tube and also provides a means to tune the cavity to the desired frequency. Prior to the initial tuning process, the internal cells were combined into subassemblies built from adjacent-AC half-cells brazed to the lower half of a TC cell. A cutaway view of this subassembly and a drift-tube ring assembly is shown in

\footnotetext{
*Work supported by US DOE, Office of Defense Programs, and the Office of Nuclear Energy, Science and Technology.

†1rybarcyk@lanl.gov
}

Figure 2. The exterior subassemblies consisted of an AC and LC half-cell brazed together. For the high-power tests, a BC cavity with a single LC half-cell was built. This $\mathrm{BC}$ cavity contains the waveguide coupling iris.

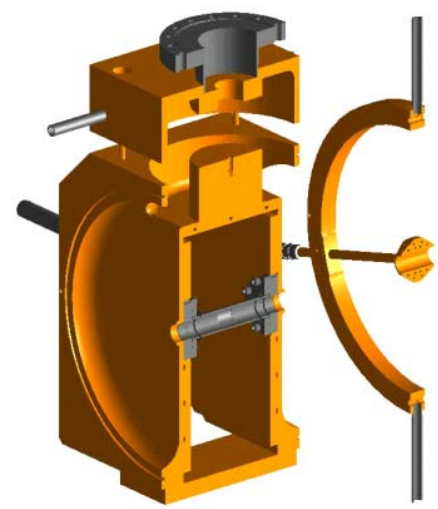

Figure 2: Cutaway view of CCDTL internal subassembly with adjacent drift-tube ring assembly. Prior to brazing, top-half of TC cell was removed for tuning cuts on upper nose.

The Section 2 cavity dimensions were determined in an iterative fashion using SUPERFISH [2] to calculate the cavity frequency and field distribution and analytical expressions for estimating the intercell coupling strength [3] and frequency effects [4] of the coupling slots. Based upon the field tilt requirements originating from beam dynamics simulations, the relative cavity locations and slot dimensions were iterated on until a solution was found. Except for the AC cell tuning ring inner diameter and the TC, LC and BC nose-to-nose gaps, all cavities were fabricated to their expected final design dimensions. Also, prior to any tuning, all the coupling slots were cut to their calculated final design dimensions. The structure was designed to operate at $700 \mathrm{MHz}$ at $90^{\circ} \mathrm{F}$.

\section{PRE-BRAZE TUNING}

During this phase of the tuning process the goal was to bring the $\pi / 2$ operating frequency, $\mathrm{f}_{\pi / 2}\left(\mathrm{vac}, 70^{\circ} \mathrm{F}\right)$, to a pre-braze target value of $700.634 \mathrm{MHz}$ and to ensure that Section 2 had the desired field distribution. The target $\mathrm{f}_{\pi / 2}$ included a $350-\mathrm{kHz}$ allowance for $\mathrm{AC}$ cell frequency shifts that were observed following the brazing of the Low Beta Hot Model CCDTL structure.

The Section 2 subassemblies were stacked with their axes along the vertical for these measurements. Several mechanical clamps were used to compress the AC-ring$\mathrm{AC}$ joints on each cell to ensure good contact and 
reasonably high "Q". The LC half cavities remained shorted while tuning Section 2. Individual cavity frequencies were measured with the nearby cells shorted with metal plungers applied in a consistent fashion across their gaps.

The mode frequencies were analyzed with DISPER using a four-parameter fit to obtain the stop band and coupling constants.

\subsection{Cavity frequency tuning}

Initially, the measured $\mathrm{AC}$ and $\mathrm{TC}$ cavity frequencies were $\sim 1-2 \mathrm{MHz}$ above and $\sim 7-8 \mathrm{MHz}$ below $700 \mathrm{MHz}$, respectively, while $\mathrm{f}_{\pi / 2}$ was $\sim 1.2 \mathrm{MHz}$ above target. For the AC cells the $\mathrm{f}_{\pi / 2}$ and individual cavity frequency data were used to determine how much to change each cavity to reach the target while minimizing the spread of individual cavity frequencies about their average.

Several iterations of tuning cuts and cavity and mode frequency measurements were performed prior to brazing. Material was removed from the tuning ring I.D. of each $\mathrm{AC}$ cavity to lower their frequency, while material was removed from the nose on the upper-half of each TC cell to raise their frequency. When $\mathrm{f}_{\pi / 2}$ was $\sim 0.6 \mathrm{MHz}$ above the target, the tuning was stopped to measure and evaluate the field distribution.

\subsection{Field Distribution}

The relative field distribution of the six AC cavities was measured using the bead-perturbation technique. A 0.125 " diameter metal sphere placed on a .006 " diameter fishing line was sent at a constant speed along the beam axis of Section 2 while the frequency shift from the nominal $f_{\pi / 2}$ was measured. The magnitude of the observed frequency shift is proportional to the square of the field strength averaged over the volume of the bead. Four measurements were averaged together for a final result.

The design field distribution calls for a linear ramp with a $+1 \%$ field tilt. The initial measurement showed an average tilt of $\sim-0.75 \%$. Both are shown in Figure 3. This field error was corrected by enlarging two of the coupling slots. To determine which slots to modify, the individual $\mathrm{AC}$ to TC couplings were first estimated by measuring the mode and individual cavity frequencies for pairs of cavities along the structure and fitting the mode frequencies with a simple coupled-circuit model. A single scale factor was applied to all the individual couplings so that a coupled-circuit model of Section 2 using these data and measured individual cavity frequencies would reproduce the measured mode spectrum and field distribution. Starting with these estimates and a simple scaling law for adjacent AC cell fields and their coupling factors, the coupling values for slots in AC cells 9 and 10 were adjusted until the calculated field distribution agreed with design. The increase in slot length was then found from the fractional change in coupling and the calculated sensitivity of coupling to slot length. Following these modifications, new beadpull data were taken. The result is in good agreement with design as shown in Figure 3.

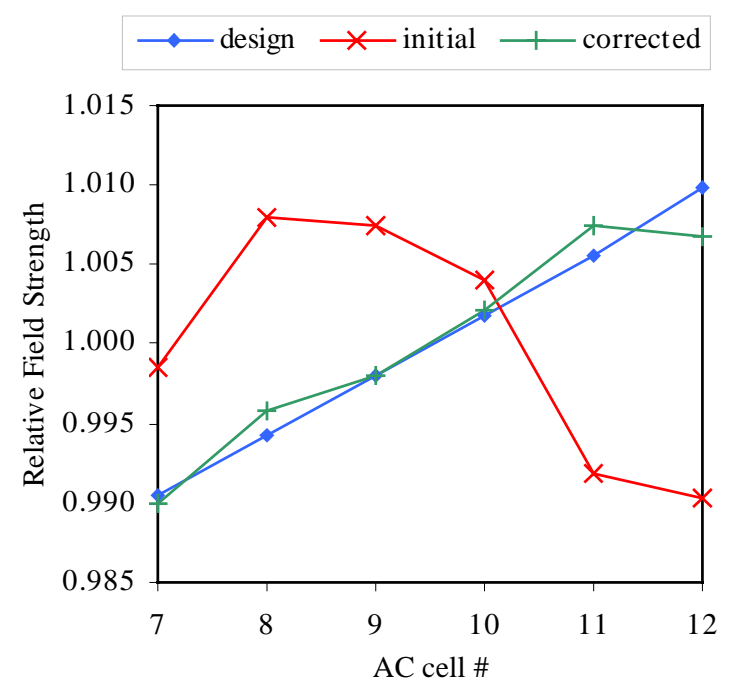

Figure 3: Section 2 relative field distributions showing comparison between initial and corrected measurements to design.

\subsection{Final pre-braze tuning}

The mode spectrum and individual cavity frequencies were measured to determine the final tuning cuts prior to braze. The average AC cell frequency was reduced by the difference between the measured and target $f_{\pi / 2}$ values. The resultant $\mathrm{f}_{\pi / 2}$ value was about $60 \mathrm{kHz}$ above the target. This was acceptable since the post-braze tuning process was to squeeze the AC cell walls to close the accelerating gap and lower it's frequency. The idea of raising the frequency of an AC cell by pulling on the endwalls had not been fully explored and so at this time was avoided. The average TC frequency was raised to produce a final stopband for the tuned-up structure of $\sim 0-50 \mathrm{kHz}$. This was done because of the difficulty envisioned in pulling the TC noses apart to raise the frequency of these cavities if the stopband ended up being well below zero. Aluminum mock BC cells were fabricated to allow the LC half-cavities on the ends of Section 2 to be tuned. The LC cells were tuned to the average of the TC cavities.

\section{POST-BRAZE DIFFERENCES}

Upon completion of the brazing process, new mode and individual cavity frequency data were taken. The TC cells showed only small (within $\pm 55 \mathrm{kHz}$ ) frequency shifts. The AC cells had mixed results. The four inner cells showed small (within $\pm 21 \mathrm{kHz}$ ) frequency shifts. The two end AC cells, 7 and 12, dropped in frequency by 395 and $515 \mathrm{kHz}$, respectively. This was ultimately associated with the endwalls of the structure being pushed in by a force that was applied by a support fixture during the brazing process.

\section{POST-BRAZE TUNING}

At this stage of the tuning process the goal was to tune the cavities to get the desired operating frequency and 
stopband, tune the BC cavity and waveguide coupling iris. With the structure now leak-tight a measurement of the operating mode frequency under vacuum was performed. From this an estimate of the frequency effects of removing the air dielectric and cavity wall deflections due to external atmospheric pressure were made. This was used to calculate a new target value for $\mathrm{f}_{\pi / 2}$.

\subsection{Cavity Tuning}

The first step was to reduce the average AC frequency to bring the $\mathrm{f}_{\pi / 2}$ to its new target. All AC cells except 12 needed to have their frequencies lowered. At this time the technique and tools for raising the frequency of an AC cell had not been developed. Therefore, it was decided to lower AC cells 7 -10 to the desire average and leave AC cell 11 high to offset AC cell 12. This solution would result in only slightly higher power in the coupling cells.

To lower the AC cell frequency, a special fixture was built. It consisted of two large plates placed against the cavity endwalls and bolted together. A boss on the surface of the each plate in the form of an arc pressed against the endwalls opposite the tuning ring. Small jackscrews near the center of the plates were then used to apply pressure to the stainless steel, beam-tube flange on the cavity walls. This technique worked extremely well. However, a small error in the $f_{\pi / 2}$ target used above resulted in a somewhat lower than expected operating frequency. This translates into an expected operating temperature of $87^{\circ} \mathrm{F}$ when the structure is at full power. This is not a problem for the high power RF tests and so it was decided not to raise the AC cavity frequencies at this time.

With the AC cells all tuned, a measurement of the mode spectrum was made with Section 2 under vacuum. The DISPER analysis showed the stopband was now $\sim 0 \mathrm{kHz}$. The TC cavities are on an independent cooling circuit and therefore can have their temperature and hence frequency adjusted separately from the rest of the structure. Therefore, it was decided not to attempt to raise their frequency by pushing the noses apart at this time.

\subsection{Bridge Coupler Cavity Tuning}

The BC cell was fabricated in two halves to facilitate tuning. To aid in setting it's frequency following brazing, a small tuning slug was added to the design. Several tuning cut iterations were made on the $\mathrm{BC}$ and adjoining LC half-cell until they were tuned to the average of the $\mathrm{AC}$ and TC cavity frequencies, respectively.

With the BC and LC cavities at their nominal frequencies, the coupling slot was cut to provide critical coupling without beam. Based upon the work of Gao [2] and cold model coupling measurements, the coupling factor was expected to scale as the slot length raised to the power $n$, where $6 \leq n \leq 7$. Initially, the exponent was estimated from the cold model data and the proportionality constant was determined from the first coupling measurement on the slot. An intermediate slot cut was made to re-evaluate the above constants and extrapolate to the final value. The final slot cut resulted in a coupling of $\sim 1.03$.

With the slot cuts completed, one final cut was made on the $\mathrm{BC}$ cell nose to bring the frequency of the cavity up to the nominal value with tuning slug penetration in the middle of the desired range. The $\mathrm{BC}$ halves were then brazed together.

Following the BC cavity braze, the tuning slug length was determined by tuning the $\mathrm{BC}$ cell frequency to minimize the power in the adjacent LC cell. This was accomplished by placing a small pick-up loop at the midplane of the LC cell and measuring the relative cavity power versus the $\mathrm{BC}$ tuning slug penetration.

\section{STATUS AND UPCOMING HIGH POWER TESTS}

Tuning of LEDA CCDTL Section 2 has been completed. It is presently being installed on the test stand in the LEDA operations building. High-power RF testing is expected to get underway sometime in June 2001. Several measurements are scheduled for this test. After the initial conditioning phase has been completed, an $\mathrm{x}^{-}$ ray endpoint measurement will be made to calibrate the cavity power, low-level RF amplitude set point with CCDTL gap voltage. Measurements to determine the adequacy of cooling on the TC cells will also be made. High power stopband measurements are also scheduled.

\section{ACKNOWLEDGEMENTS}

The authors would like to acknowledge the efforts of $\mathrm{H}$. Haagenstad, F. Martinez, J. Mitchell, D. Montoya, A. Rendon, J. Szalczinger and T. Wright of the LANSCE-1 mechanical section for their efforts in fabricating, assembling, testing and finally preparing this structure for high power tests. We also thank F. Sigler of the LANSCE-1 design section for his outstanding efforts in helping us to reach our goal. One of us (LJR) would especially like to thank L. Young and J. Billen for numerous discussions and suggestions.

\section{REFERENCES}

[1] J.H. Billen, et. al., "A New RF Structure for Intermediate-Velocity Particles”, Proc. 1994 Int. Linac Conf., Tsukuba, Japan, (1994) 341-345.

[2] J.H. Billen and L.M. Young, "POISSON/SUPERFISH on PC Compatibles," Proc. 1993 Particle Accelerator Conference, Wash. D.C., (1993) 790-792.

[3] J. Gao, "Analytical formula for coupling coefficient $\beta$ of a cavity-waveguide coupling system", Nucl. Instr. Meth. A309 (1991) 5-10.

[4] J. Gao, "Analytical formulas for the resonant frequency changes due to opening apertures on cavity walls", Nucl. Instr. Meth. A311 (1992) 437-443. 